\title{
Pharmacokinetics of Dantrolene in the CVVH/PE Treatment of Malignant Hyperthermia in a 14-year-old Chinese Boy: A Case Report and Literature Review
}

Xiao-Xiao LI ${ }^{1}$, Chao $\mathrm{Li}^{2}$, Yang Zhou ${ }^{2}$, Xin Xiong ${ }^{2}$, Chuhui Wang ${ }^{2}$, Congya Zhou ${ }^{2}$, Bin $\mathrm{Han}^{2}, \mathrm{Li}_{\mathrm{Yang}}{ }^{2}$, and Xiangyang Guo ${ }^{2}$

${ }^{1}$ Affiliation not available

${ }^{2}$ Peking University Third Hospital

August 8, 2021

\begin{abstract}
Malignant hyperthermia $(\mathrm{MH})$ is a rare life-threatening response that triggered by exposure to specific anesthetics commonly used during surgery interventions. Dantrolene is a well-known drug which used as first-line therapy for MH. A 14-year-old Chinese boy with a mutation in RYR1 whose muscle biopsy diagnosis was central core disease (CCD) occurred MH after cervical spine surgery underwent general anesthesia without volatile anesthetics or succinylcholine. The MH crisis treatment workflow was started and intravenous dantrolene was used soon which combined with sequent continuous veno-venous hemofiltration $(\mathrm{CVVH})$ and plasma exchange (PE) therapy. We explored the pharmacokinetics of dantrolene during the treatment and the results showed that a one-compartment model with first-order absorption and elimination was sufficient to characterize dantrolene pharmacokinetics. The clearance estimate for dantrolene was $0.43 \mathrm{~mL} /\left(\mathrm{min}^{*} \mathrm{~kg}\right)$ and volume of distribution was 0.61 $\mathrm{L} / \mathrm{kg}$. The clearance of dantrolene was significantly affected by CVVH and PE.
\end{abstract}

Pharmacokinetics of Dantrolene in the CVVH/PE Treatment of Malignant Hyperthermia in a 14-year-old Chinese Boy: A Case Report and Literature Review

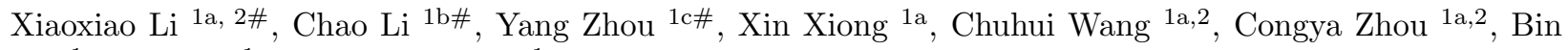
$\mathrm{Han}^{1 \mathrm{c}}$, Li Yang ${ }^{1 \mathrm{a} *}$, Xiangyang Guo ${ }^{1 \mathrm{c} *}$

1a Department of Pharmacy, b Department of Critical Care, c Department of Anesthesiology, Peking University Third Hospital, Beijing, China

2 Department of Pharmacy Administration and Clinical Pharmacy School of Pharmaceutical Sciences, Peking University, Beijing, China

\# Xiaoxiao Li, Chao Li, and Yang Zhou contributed equally to this work

* Corresponding authors:

Li Yang M.Sc.,

Department of Pharmacy, Peking University Third Hospital

Add: 49 North Garden Rd., Haidian District Beijing 100191, P.R.China

Telephone: 86-10-82265743

Email: lilianyangli@163.com 
Xiangyang Guo M.D.,

Department of Anesthesiology, Peking University Third Hospital

Add:49 North Garden Rd., Haidian District Beijing 100191, P.R.China

Telephone: 86-10-82267276

Email: puthmzk@hsc.pku.edu.cn

Abstract: Malignant hyperthermia $(\mathrm{MH})$ is a rare life-threatening response that triggered by exposure to specific anesthetics commonly used during surgery interventions. Dantrolene is a well-known drug which used as first-line therapy for MH. A 14-year-old Chinese boy with a mutation in RYR1 whose muscle biopsy diagnosis was central core disease (CCD) occurred $\mathrm{MH}$ after cervical spine surgery underwent general anesthesia without volatile anesthetics or succinylcholine. The MH crisis treatment workflow was started and intravenous dantrolene was used soon which combined with sequent continuous veno-venous hemofiltration $(\mathrm{CVVH})$ and plasma exchange (PE) therapy. We explored the pharmacokinetics of dantrolene during the treatment and the results showed that a one-compartment model with first-order absorption and elimination was sufficient to characterize dantrolene pharmacokinetics. The clearance estimate for dantrolene was $0.43 \mathrm{~mL} /\left(\mathrm{min}^{*} \mathrm{~kg}\right)$ and volume of distribution was $0.61 \mathrm{~L} / \mathrm{kg}$. The clearance of dantrolene was significantly affected by CVVH and PE.

Keywords: malignant hyperthermia $(\mathrm{MH})$; dantrolene; pharmacokinetics; continuous veno-venous hemofiltration $(\mathrm{CVVH})$; plasma exchange $(\mathrm{PE})$

\section{Introduction}

Malignant hyperthermia $(\mathrm{MH})$ is a rare life-threatening response that occurs in genetically susceptible individuals that triggered by exposure to halogenated/volatile anesthetics commonly used during surgery interventions, characterized by muscle rigidity, hyperthermia, rhabdomyolysis, acidosis, tachycardia, and, if untreated, death(1). Dantrolene is a well-known inhibitor of $\mathrm{Ca}^{2+}$ release in skeletal muscle that has been used clinically as first-line therapy for $\mathrm{MH}(2)$. A 14-year-old Chinese boy occurred fulminant $\mathrm{MH}$ after cervical spine surgery even not exposed to volatile anesthetics or succinylcholine and was successfully cured by combined treatment of intravenous dantrolene, continuous veno-venous hemofiltration (CVVH), and plasma exchange (PE) therapy. In this case, the pharmacokinetics of intravenous dantrolene with $\mathrm{CVVH} / \mathrm{PE}$ during fulminant malignant hyperthermia were explored. We present the following case in accordance with the CARE reporting checklist.

\section{Case Presentation}

A 14-year-old boy with a 7-year history of progressively worsening cervical lordosis deformity was hospitalized and planned for elective cervical spine surgery. His muscle biopsy was performed and the pathological diagnosis was central core disease (CCD). Subsequent genetic testing revealed mutations in RYR1(NM $001042723)$ c. $11120 \mathrm{~A}>\mathrm{G}$ and c.12227C $>\mathrm{T}$ (figure 1).

On presentation, this 42 -kg-weight patient was afebrile with normal vital signs, while his laboratory tests, ECG, and Chest X-ray were within normal limits. He later underwent posterior cervical C3-T3 soft tissue release, pedical screw internal fixation and fusion, and excessive lordosis deformity. Owing to his mutations encoding for abnormal RYR1 receptors, total intravenous anesthesia with propofol and remifentanil was used throughout the operation. Neither volatile anesthetic agents nor succinylcholine was administered during the uneventful surgical procedure. The intraoperative plasma CK, CK-MB, and myoglobin were all within the normal range. The patient was transferred to the intensive care unit (ICU) after surgery for further observation.

The patient arrived in ICU and routine monitors were applied, showing normal vital signs and body temperature: $\mathrm{BP} 110 / 70 \mathrm{mmHg}$, $\mathrm{HR} 50 \sim 60 \mathrm{bpm}, \mathrm{SpO}_{2}$ 100\%, axillary temperature 35.0. Analgesia-sedation during the mechanical ventilation was maintained with propofol $\left(1^{\sim} 1.5 \mathrm{mg} / \mathrm{kg}\right)$ and butorphanol $\left(0.2^{\sim} 0.25\right.$ 
$\mathrm{mg} / \mathrm{h}$ ). After 90 minutes of mechanical ventilation, the patient developed a sudden onset sinus tachycardia of $140 \sim 160 \mathrm{bpm}$ while his arterial blood pressure increased to $120 \sim 130 / 70^{\sim} 80 \mathrm{mmHg}$. The patient began to appear with masseter muscle rigidity (MMR) and generalized muscle rigidity intermittently, accompanied by an increase in axillary temperature from 36.0 to 39.4. Arterial blood gases showed mixed metabolic and respiratory acidosis with hyperkalemia $\left(\mathrm{pH} 7.236, \mathrm{PaCO}_{2} 52.3 \mathrm{mmHg}, \mathrm{K}^{+} 4.38 \mathrm{mmHg}\right.$, Lac $\left.5.7 \mathrm{mmol} / \mathrm{L}\right)$. A clinical diagnosis of fulminant $\mathrm{MH}$ was made according to the clinical grading scale (CGS) (3).

The MH crisis treatment workflow was started immediately and dantrolene sodium (NDC 42023-123-06, Par Pharmaceutical Companies, Inc., USA) was administered at the initial dose of $2.5 \mathrm{mg} / \mathrm{kg}$ which was followed by a maintenance dose of $0.25 \mathrm{mg} / \mathrm{kg} / \mathrm{h}$ (Supplementary Table 1). The inspired oxygen fraction increased to $100 \%$ immediately, meanwhile increased respiratory rate and tidal volume to maximize ventilation and lower the $\mathrm{PaCO}_{2}$. Physical cooling was conducted including an ice cap as well as a reducing the temperature carpet. Then $\mathrm{PaCO}_{2}$ declined to $28.7 \mathrm{mmHg}$ and the axillary temperature dropped to 38.1. However, the status of the patient deteriorated over the next 24 hours, MMR could still be observed intermittently, with creatine phosphokinase $(\mathrm{CK})$, serum myoglobin $(\mathrm{Mb})$, urine myoglobin $(\mathrm{UMb})$, and lactate dehydrogenase (LDH) increasing progressively. Maximal plasma concentrations of CK and Mb were $3303 \mathrm{IU} / \mathrm{L}$ and 579 $\mathrm{ng} / \mathrm{mL}$, respectively. Accordingly, the diagnosis of rhabdomyolysis syndrome was ascertained for the patient. Continuous veno-venous hemofiltration $(\mathrm{CVVH})$ was then initiated, 17 hours after the start of the event.

Analgesia-sedation treatments, bedside CVVH (AQUARIUS, Nikkiso Europe, Langenhagen, Germany), the hemofilter (HF1200) was a $1.25 \mathrm{~m}^{2}$ glycerin-free polysulfone-membrane Polyflux (Medivators, Minnesota, USA). The replacement solution was a bicarbonate-based solution containing $1.91 \mathrm{mg} / \mathrm{ml}$ glucose and 0.02 $\mathrm{mg} / \mathrm{ml}$ magnesium, while the predilution and postdilution rates were both $1000 \mathrm{ml} / \mathrm{h}$. Blood flow and fluid loss rates were $180 \mathrm{~mL} / \mathrm{min}$ and $200 \mathrm{ml} / \mathrm{h}$, respectively. The rectification of acid intoxication, urine alkalization, as well as several supportive treatments was performed at the same time and dantrolene sodium was discontinued. After administered CVVH for nearly 36 hours, the values of CK, Mb, and LDH decrease slowly, while his muscle tremors still occurred intermittently throughout those continuous treatments mentioned above. Due to the poor effect during CRRT, his rhabdomyolysis syndrome was treated with plasma exchange (PE). The plasma filter was a $0.6 \mathrm{~m}^{2}$ polyurethane-membrane Polyflux (Fresenius, Bad Homburg, Germany). Plasma rate and total volumes were $1000 \mathrm{~mL} / \mathrm{min}$ and $3000 \mathrm{ml}$, respectively.

Artery blood samples were collected before and after PE and followed by the blood samples collected after dantrolene administration for up to $12 \mathrm{~h}$ (at minutes $0,15,30,60,120,240,360$ for the first bolus as 2.5 $\mathrm{mg} / \mathrm{kg}$ dantrolene, and at minutes 0, 30, 120, 240, 360 for the second bolus as $1 \mathrm{mg} / \mathrm{kg}$ dantrolene). Plasma was separated by centrifuge and stored at -80 until assayed.

The plasma concentrations of dantrolene were measured simultaneously by using a validated highperformance liquid chromatography (Shimadzu LC-30A; Shimadzu, JP) coupled with tandem mass spectrometry (ABSciex $4000^{+}$triple quadrupole system, ABSciex Corp., USA) assay method. Data were acquired and processed by Analyst 1.6.2 software (ABSciex Corp.). A 50 $\mu$ L plasma sample was extracted by protein precipitation.

A reported population PK model, with the parameters from healthy adults, was applied for predicting dantrolene plasma concentrations (4). For all calculations, NONMEM software (version 7.3.0, ICON Development Solutions) was used. However, the predictions matched with the measured values only nearby the lower limit, suggesting new pharmacokinetic parameters for dantrolene should be developed, which might be fit for adolescents with PE/ CVVH (Figure 2). Thus, PK analysis was performed by using a nonlinear mixed-effects model. Data were analyzed using a first-order conditional estimation method (5). One- and two-compartment models with first-order absorption and linear elimination were investigated to determine the optimal structural model. The clearance (CL) and volume of distribution (Vd) of dantrolene were characterized and estimated. Because we only have one patient, the inter-individual variability was fixed as 0 and no covariate was retained in the final model.

A one-compartment model with first-order absorption and elimination was sufficient to characterize dantro- 
lene pharmacokinetics. The parameter estimate for dantrolene CL was $0.43 \mathrm{~mL} /\left(\mathrm{min}^{*} \mathrm{~kg}\right)$ and for $\mathrm{Vd}$ was $0.61 \mathrm{~L} / \mathrm{kg}$, and Table 1, Figure 3 shows the details. The CL was significantly affected by CVVH and PE.

\section{Discussion}

$\mathrm{MH}$ is a well-known but potentially lethal disorder that is triggered by volatile anesthetics and depolarizing muscle relaxants. Most of the patients who suffered MH were reported having a genetic defect in the RYR1 gene which was associated with disordered calcium channel function and disease. This adolescent patient we reported was also diagnosed as CCD with RYR1 mutations and occurred MH postoperatively even absence of volatile anesthetics and depolarizing muscle relaxants. Dantrolene is the only clinically available agent for the specific treatment and is established to decrease the mortality of $\mathrm{MH}$ to $<10 \%(2)$. To monitor the effectiveness and safety of intravenous dantrolene during fulminant malignant hyperthermia, the blood samples were collected and plasma concentrations of dantrolene were measured.

As an orphan drug, studies focusing on PK parameters of dantrolene injection were limited worldwide. Besides, a gap existed between the enrolled volunteers/ patients and our patients both in the demographic characteristics and physical status, making the generalizability border of available data vague. There was only one population PK model reported previously, by assuming one single compartment, the parameters were from $27 \pm 5 \mathrm{yr}$ old, weighted $70 \pm 12 \mathrm{~kg}$ healthy adults (4). However, the simulated dantrolene plasma concentrations for our adolescent patient (14-year-old, 48kg) showed poor goodness of fit compared with the measured ones. Also, the parameters using the non-compartmental method from another study were calculated from $3.6 \pm 51.3 \mathrm{yr}$ old, weighted $17.4 \pm 4.9 \mathrm{~kg}$ malignant hyperthermia susceptible children during anesthesia(6).

The pharmacokinetic parameters, i.e., CL only based on renal clearance, for dantrolene administration in this adolescent patient presenting as $0.33 \mathrm{~mL} /\left(\mathrm{min}^{*} \mathrm{~kg}\right)$ is lower to those reported previously both in healthy adults $\left(0.43 \pm 0.043 \mathrm{~mL} /\left(\mathrm{min}^{*} \mathrm{~kg}\right)(4)\right.$ and in malignant hyperthermia susceptible children $(0.64 \pm 0.18$ $\mathrm{mL} /\left(\min ^{*} \mathrm{~kg}\right)(6)$. Because of the high myoglobin concentrations, an acute kidney injury during malignant hyperthermia attack might occur, followed by a slow elimination of dantrolene. Moreover, a coefficient of variation might lie in the methods for the measurement of dantrolene among different studies.

Owing to the patient's complicated with massive rhabdomyolysis, early institution of CVVH even PE seems important(7). However, until now, there was no recommendation about how to adjust the dosage of dantrolene during and after $\mathrm{CVVH} / \mathrm{PE}$, and to our knowledge. This case is the first one to explore the pharmacodynamics during $\mathrm{CVVH} / \mathrm{PE}$. Although CL increased from $0.33 \mathrm{~mL} /\left(\mathrm{min}^{*} \mathrm{~kg}\right)$ to $0.42 \mathrm{~mL} /\left(\mathrm{min}^{*} \mathrm{~kg}\right)$ during the PE period and even elevated to $0.51 \mathrm{~mL} /\left(\mathrm{min}^{*} \mathrm{~kg}\right)$ during $\mathrm{CVVH}$ and PE period, considering the safety and cost-effectiveness, together with the accumulation of dantrolene, a loading dose of $2.5 \mathrm{mg} / \mathrm{kg}$ dantrolene after PE period seems unnecessary. Further studies are needed to explore either a safe accumulated dose limit for avoiding dantrolene-induced cardiotoxicity or the mechanism of the toxicity.

\section{Ethical Statement}

The authors are accountable for all aspects of the work in ensuring that questions related to the accuracy or integrity of any part of the work are appropriately investigated and resolved. All procedures performed in studies involving human participants were in accordance with the ethical standards of the institutional and/or national research committee(s) and with the Helsinki Declaration (as revised in 2013). Written informed consent was obtained from the patient.

\section{Acknowledgments}

We thank ICU nurses (Department of Critical Care, Peking University Third Hospital) and Yi Chen M.Sc. (Department of Pharmacy Administration and Clinical Pharmacy School of Pharmaceutical Sciences, Peking University) for the help of the arterial blood sampling. We thank Professor Yanping Deng Ph.D. (National Institution on Drug dependence, Peking University) for the help in calculating PK parameters. We thank Livzon Pharm for offering dantrolene (Revonto) for free, together with the method of sample preparation and storage. 


\section{Contributions}

$\mathrm{X}-\mathrm{x} \mathrm{Li}$ was responsible for designing sampling strategies, collecting samples, calculating PK parameters, and drafting the manuscript. C Li was responsible for the treatment decision, collecting clinical data, and involved in drafting the manuscript. Y Zhou and B Han were responsible for offering clinical data and revising the manuscript. X Xiong was responsible for establishing a measuring method of plasma concentrations. C-h Wang was involved in calculating PK parameters and revising the manuscript. C-y Zhou was responsible for the plasma concentrations of dantrolene determination. L Yang was responsible for the access to dantrolene, checking sampling strategies and measured data, and approving the final version of the manuscript. X-y Guo was responsible for the treatment decision, the access to dantrolene, getting informed consent from parents, and approving the final version of the manuscript.

\section{References}

1. Hopkins PM, Girard T, Dalay S, et al. Malignant hyperthermia 2020: Guideline from the Association of Anaesthetists. Anaesthesia 2021;76:655-64.

2. Krause T, Gerbershagen MU, Fiege M, et al. Dantrolene-a review of its pharmacology, therapeutic use and new developments. Anaesthesia 2004;59:364-73.

3. Larach MG, Localio AR, Allen GC, et al. A clinical grading scale to predict malignant hyperthermia susceptibility. Anesthesiology 1994;80:771-9.

4. Podranski T, Bouillon T, Schumacher PM, et al. Compartmental pharmacokinetics of dantrolene in adults: do malignant hyperthermia association dosing guidelines work? Anesth Analg 2005;101:1695-9.

5. Hooker AC, Staatz CE, Karlsson MO. Conditional weighted residuals (CWRES): a model diagnostic for the FOCE method. Pharm Res 2007;24:2187-97.

6. Lerman J, McLeod ME, Strong HA. Pharmacokinetics of intravenous dantrolene in children. Anesthesiology 1989;70:625-9.

7. Schenk MR, Beck DH, Nolte M, et al. Continuous veno-venous hemofiltration for the immediate management of massive rhabdomyolysis after fulminant malignant hyperthermia in a bodybuilder. Anesthesiology 2001;94:1139-41.

Table1 Pharmacokinetic parameters estimated for dantrolene. (A) during PE period, (B) during CVVH and PE period, (C) after PE, only with renal clearance, (D) the whole course as (B) $+(\mathrm{C})$

\begin{tabular}{lll}
\hline Period & CL, $\mathrm{mL} /\left(\mathrm{min}^{*} \mathrm{~kg}\right)$ & $\mathrm{V}, \mathrm{L} / \mathrm{kg}$ \\
\hline (A) & 0.42 & 0.38 \\
(B) & 0.51 & 0.48 \\
(C) & 0.33 & 0.50 \\
(D) & 0.43 & 0.61 \\
\hline
\end{tabular}

\section{FIGURE LEGENDS}

Figure 1. Family genogram for RYR1 (NM 001042723) mutations. The patient's mother and third sister were confirmed to have homologous gene mutations, while his father as well as eldest sister and second sister did not find the same mutations in genetic testing.

Fig.2 The goodness of fit between predicted and measured dantrolene plasma concentration (ng/ml). According to the United States MHAUS guidelines (the first bolus as $2.5 \mathrm{mg} / \mathrm{kg}$ dantrolene, and $1 \mathrm{mg} / \mathrm{kg}$ bolus every $6 \mathrm{~h}$ then), predicted concentrations were calculated with the reported population PK model (4) and didn't match well with the measured concentrations over the entire concertation range after PE. 
Fig 3. Measured dantrolene plasma concentration (dots) during CVVH, PE, and renal clearance period.
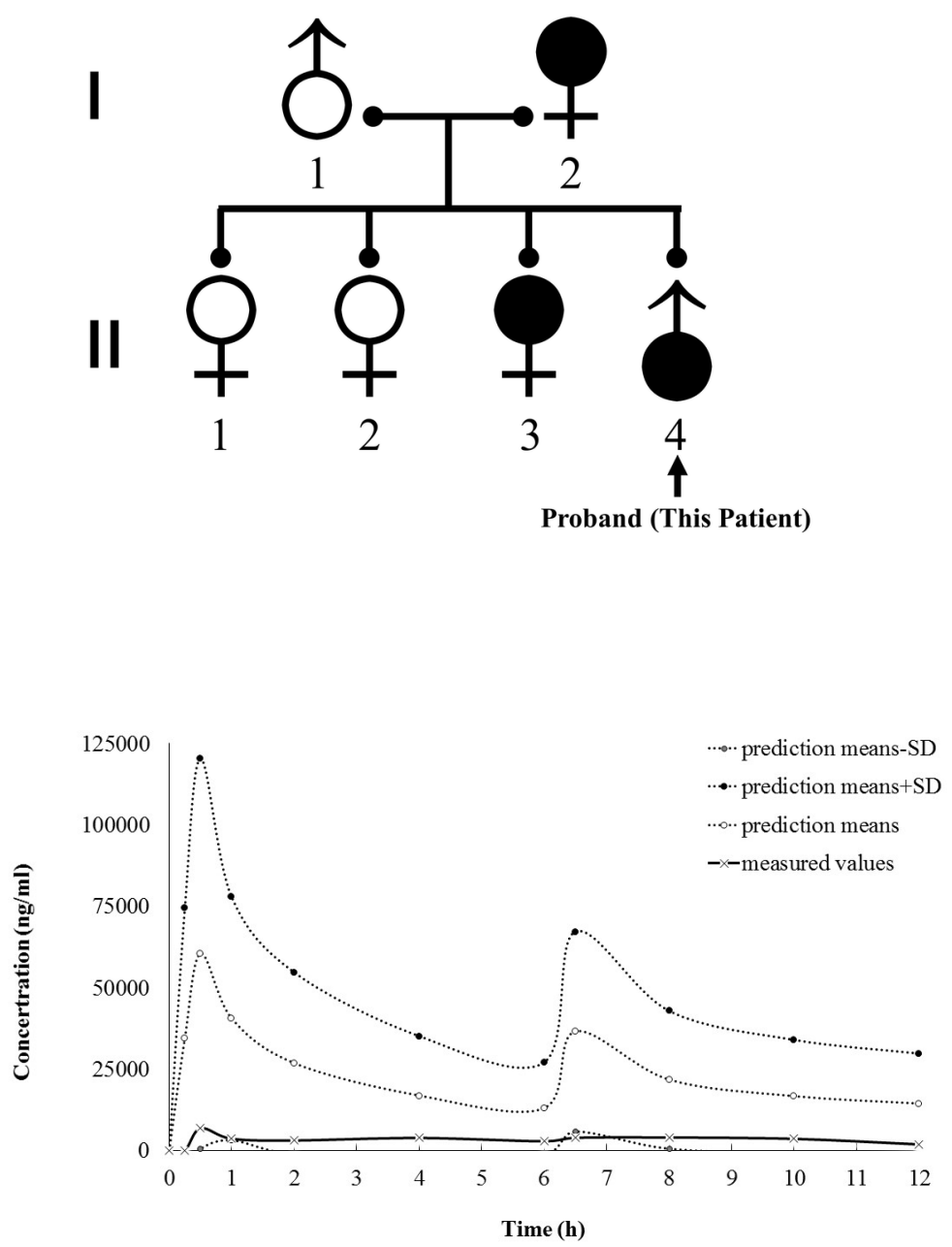


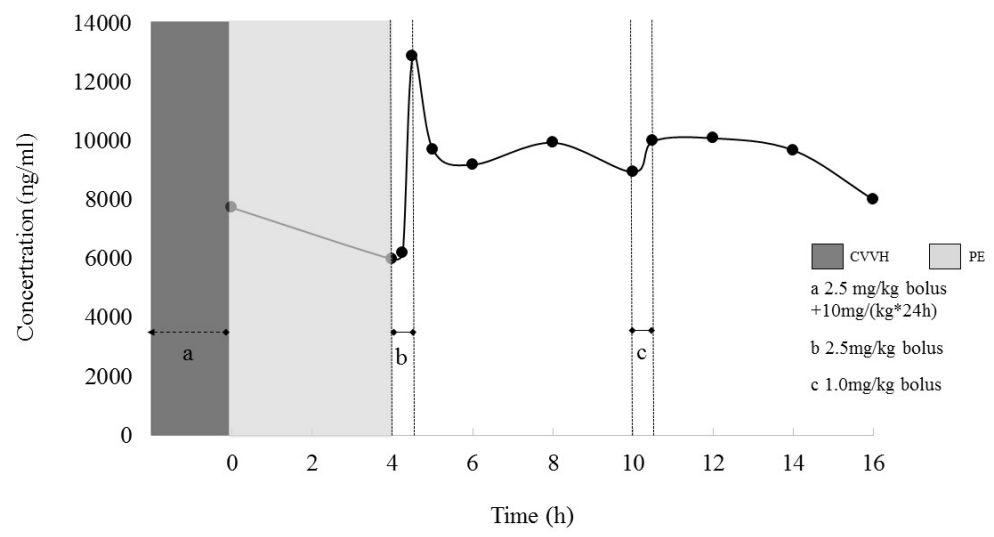

